\title{
A Survey Course on VEE Pro Software for Engineers and Technologists
}

\author{
Thomas E. Hulbert, Robert B. Angus \\ Northeastern University \\ Boston, MA 02115-5096
}

\begin{abstract}
Data acquisition requirements for manufacturing, development, and research applications have grown significantly. A continued need is predicted for standards and software to efficiently process and store that data.

The authors, who created Just-in-Time (JIT) Education ${ }^{\mathrm{TM}}$, were invited to meet with a multinational, high-technology company. Their corporate team identified a critical need for training technicians and designers on VEE Pro as the standard software to simulate, and run data acquisition processes. The program was developed to train their technicians and designers, and then the engineering staff. This paper will focus on the engineers' course - the design of the course, the results, and its future direction.
\end{abstract}

The curriculum developers, using JIT Education concepts, developed the twelve-hour overview course. Faculty-developed labs used in the technician course are provided; participants analyze and evaluate the labs back to the beginning of those laboratories.

The objectives were to examine the programs at the icon level. At the conclusion of the course, the participants ask the "what if" questions. This has enabled the entire technical staff (engineers, technologists, and technicians) to develop improved data acquisition applications and experiments.

Eight participants have taken the course and provided feedback via a questionnaire. Lessons learned from this experience can be applied to a course for engineering technology students and the improvement of the engineers' course.

\section{Introduction}

This paper outlines and presents the results of the development and teaching of a unique course. A corporate client of Northeastern University had contracted to teach their technicians and technologists a testing and measurement software package known as VEE. This was originally marketed by Hewlett Packard (HP). It is now a product of the recently formed spin-off company: Agilent Technologies. 
The client began the training effort using a bottom up approach training by the "user" level first. Over sixty technicians have been trained but only several engineers are knowledgeable of the software and its capability. Results of the initial effort were reported at last year's conference ${ }^{3}$. This course is designed as an introduction and survey of the software for engineers and supervisors.

This paper begins with a summary taken from the companies' Web pages of three major software packages: Visual Basic, LabView, and VEE. The next section is a review of JIT Education that was the foundation for the development of the courses. The paper continues with a review of the application of JIT Education and an overview of VEE.

The development of the "Inside the Icons" Labs for engineers is described, and a summary of the evaluation of the first offering is presented. The information and data from all of the development and teaching were used to postulate a methodology for delivery of these laboratories for undergraduate engineering and technology students.

\section{Comparison of VEE, LabView, and Visual Basic}

A qualitative summary of the three major software packages used for Test and Measurement applications is included below. The authors did not have market data available, but their experiences and that of others in the business indicate that the three of the major products are Visual Basic, LabView, and Agilent VEE. The following summary is taken from the respective company's literature on the Internet and presented for comparison in this paper. The authors feature VEE in this paper as they are trainers in this software. The comparisons follow:

VEE Pro (Agilent) Web Address: www.agilent com

Product Highlights and Overview: Agilent Technologies' vision is to connect your measurements to minds to gain insight, so you can get results to your market, using de-facto industry standards wherever possible.

Introducing an expanded Agilent VEE 6.0 software family, which builds on HP VEE's measurement expertise with its unprecedented 'measurements to minds' capability such as advanced math visualization and signal processing. The Agilent VEE software is compelling and use in the graphical programming environment delivers fast measurement analysis results.

Features:

Delivers measurement analysis leadership

MathWorks MATLAB script and signal processing embedded

Smart measurements

- take any measurement and control; any PC card or instrument from any vendor

- utilize over 500 National Instruments LabWindows/CVI drivers

Proceedings of the 2001 American Society for Engineering Education Annual Conference \& Exposition Copyright 2001, American Society for Engineering Education 
- embedded I/O configuration for easier unlimited run time distribution

- program the properties of your instruments

- automatically verify instrument addresses and other parameters at runtime

- automatic error troubleshooting for instrument time-outs

Standards friendly

- complement VEE with the best capabilities of other languages

- use VEE's powerful instrument discovery and measurement expertise in Visual Basic or C/C++ via ActiveX

- utilize Word reports, Excel spreadsheets, capabilities, Outlook paging/email, and Access database functions in VEE

- programs and graphics can be monitored from the Web; remote diagnostics

- improved ISA, PCI, CPCI, PCMCIA support

LabVIEW (National Instruments) Web Address: www.ni com

Product Highlights and Overview: LabVIEW is a high productivity graphical programming language for building instrumentation systems. With LabVIEW, you can quickly create front panel user interfaces, giving you interactive control of your software system. To specify the functionality, you intuitively assemble block diagrams, a natural design notation for engineers and scientists. LabVIEW has all of the same development tools and language capabilities of a standard language such as C- looping and case structures, configuration management tools, and compiled performance.

Features:

- Has graphical programming, TestStand Test executive software, Measurement Studio for Visual C++, Visual Basic, and C (LabWindows/CVI)

- Also has turnkey and configuration tools (Measure) and ready-to-run applications (Virtual Bench and Bio Bench)

- Gets job done quickly with integrated solutions, interactive tools, and wizards/code generation

- Benefits are

rapid application development

tight integration of software with a wide range of hardware

high performance compiled execution

lower total cost of ownership with long-term software compatibility and

flexibility with emerging computer and instrumentation technologies

Visual Basic (VB) (Microsoft) Web Address: www.microsoft com/vbasic Product Highlights and Overview: VB development system is the most productive tool for creating fast business solutions for Windows and the Web. A comprehensive, rapid application development environment helps developers quickly create and deploy client/server applications, plus easily program for the Internet using familiar VB programming tools and techniques. 
Features:

Tap into productivity and performance by

- programming with the world's most popular programming language to quickly create high-performance applications and components in a RAD environment

- building fast, native code applications and components that use the technology in Visual C++® development system

- making applications and components thread-safe without manual thread management

- increasing development speed and productivity with Intelligence® technology

- creating a broad range of reusable COM-based components including ActiveX® controls as well as middle-tier and server-side components

- $\quad$ using VB skills in Microsoft Office suite

Create data-centric applications

Increase efficiency with integrated Visual Database tools

Start building Internet applications today

\section{A Review of JIT Concepts}

The Just-In-Time Education ${ }^{\mathrm{TM}}$ concept is drawn from manufacturing. In curriculum design, material to be taught is presented when it is needed to tie to the mainstream of learning ${ }^{3,4}$. The material is divided into learning objectives that have measurable inputs and outputs ${ }^{6}$. These objectives are joined in a networked-precedence diagram to guide the developers to present the material when required.

JIT includes all components of educational practice: curriculum, instruction, assessment, and teaching. The curriculum is comprised of an integrated sequence of nodes and related topics that incorporate competencies.

Educational change occurs at the node (Just-in-Time where the mathematics, physics, technologies, and related competencies are presented to the student). The content of a node is similar to the description of a topic within a course but at a more detailed level; it is presented in behavioral terms. Nodes introduce relevant knowledge, skills, and attitudes via combinations of lecture, computer simulation, written documents or other traditional or modern methodology. Student participation in these related activities reinforces their competencies and forms the basis for student assessment.

Nodes are then linked as concept charts (networks) where the outcomes of the previous node become the input to the next node. From the sequence of Nodal Concept Charts, a Unified Concept Chart (Figure 1) is developed that documents the integration of nodes, units, disciplines, and student competencies. Overall Unified Concept Charts for the sixty hour technician-training program systematically were organized the curriculum and identified entry and exit points along the sequence of nodes. The flexibility of node-based curriculum makes it possible to develop alternative curriculum designs by relating the nodes to employer needs ${ }^{4}$. 
The development of the Nodal Concept Chart illustrates how mathematics, physics, and engineering economics nodes flow into the technology node. (The Nodal Concept Chart later becomes a portion of a Unified Concept Chart.) The math node is preceded by those nodes required to achieve this level of competency $(\mathrm{x}=\mathrm{yz})$. The physics and technology nodes have similar competencies that have to be achieved first. Upon successful completion and assessment of applying $\mathrm{x}=\mathrm{yz}$ to Hooke's Law $(\mathrm{f}=\mathrm{kd}$ ) at the physics node, the technology application is next. Upon successful completion of the beam deflection experiment, the product description(s) are then synthesized and documented. The results are then assessed by the faculty.

The students now branch to the physics application illustrated by the application of Ohm's Law, $\mathrm{e}=\mathrm{Ri}$ (electrical technology). They study resistance and how resistors are applied in electrical circuits. The Unified Concept Chart can also include other layers (such as flow of fluids and transfer of heat energy) based on this math node.

The sequence of competencies is documented via Unified Concept Charts. These charts interlock nodes, units, and disciplines. They also provide a basis for systematically organizing the curriculum and sequencing the material to be presented.

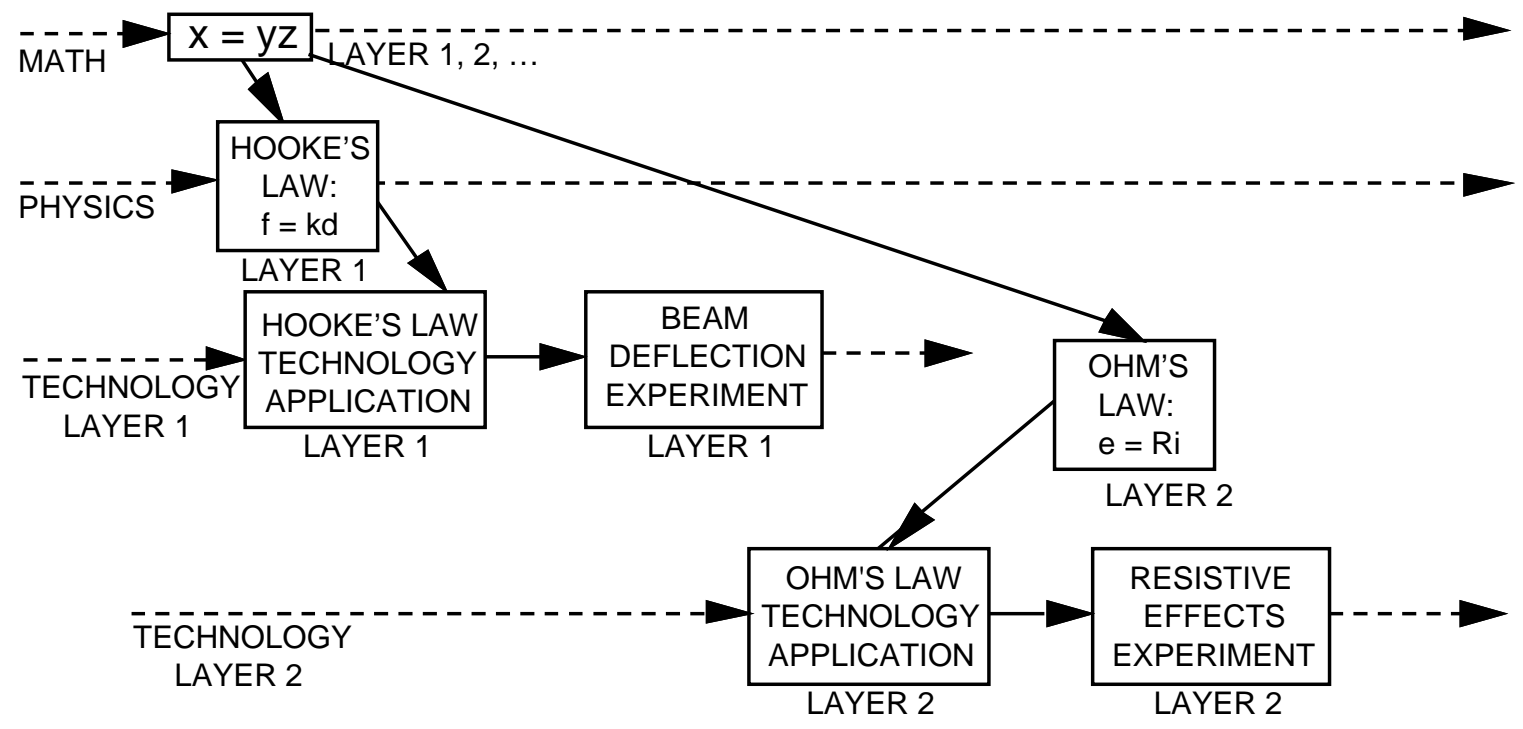

Figure 1 Unified Concept Chart

\section{VEE Pro Overview}

VEE (Virtual Engineering Environment) is a visual, mostly intuitive, programming language that dramatically reduces test preparation and development time ${ }^{3}$. Programs can be written for data acquisition, data processing, and process control.

Proceedings of the 2001 American Society for Engineering Education Annual Conference \& Exposition Copyright 2001, American Society for Engineering Education 
Programs are prepared by connecting icons together on the computer screen. The resulting program resembles a block diagram that runs like textual language programs. Virtual instruments exist within the program. Real (actual) instruments must be attached via special plug-in cards; they often require a separate card for each instrument.

VEE Pro programs control in both directions. One can control virtual or actual instruments via VEE Pro, what is to be monitored, how data is processed and stored, and how data are processed for reports.

\section{How the "Inside the Icons" Labs were Developed}

The 12-hour course is uniquely designed for Engineers and Managers to understand the capabilities and constraints of Agilent VEE Pro. The course meets in a PC laboratory. Participants explore VEE Pro through dissecting and discovering how working VEE-based systems operate. Each lab consists of presenting VEE Pro programs at the icon level. See Figure 2 for an example of a program with the icons closed. This program graphically presents the flow of the process.

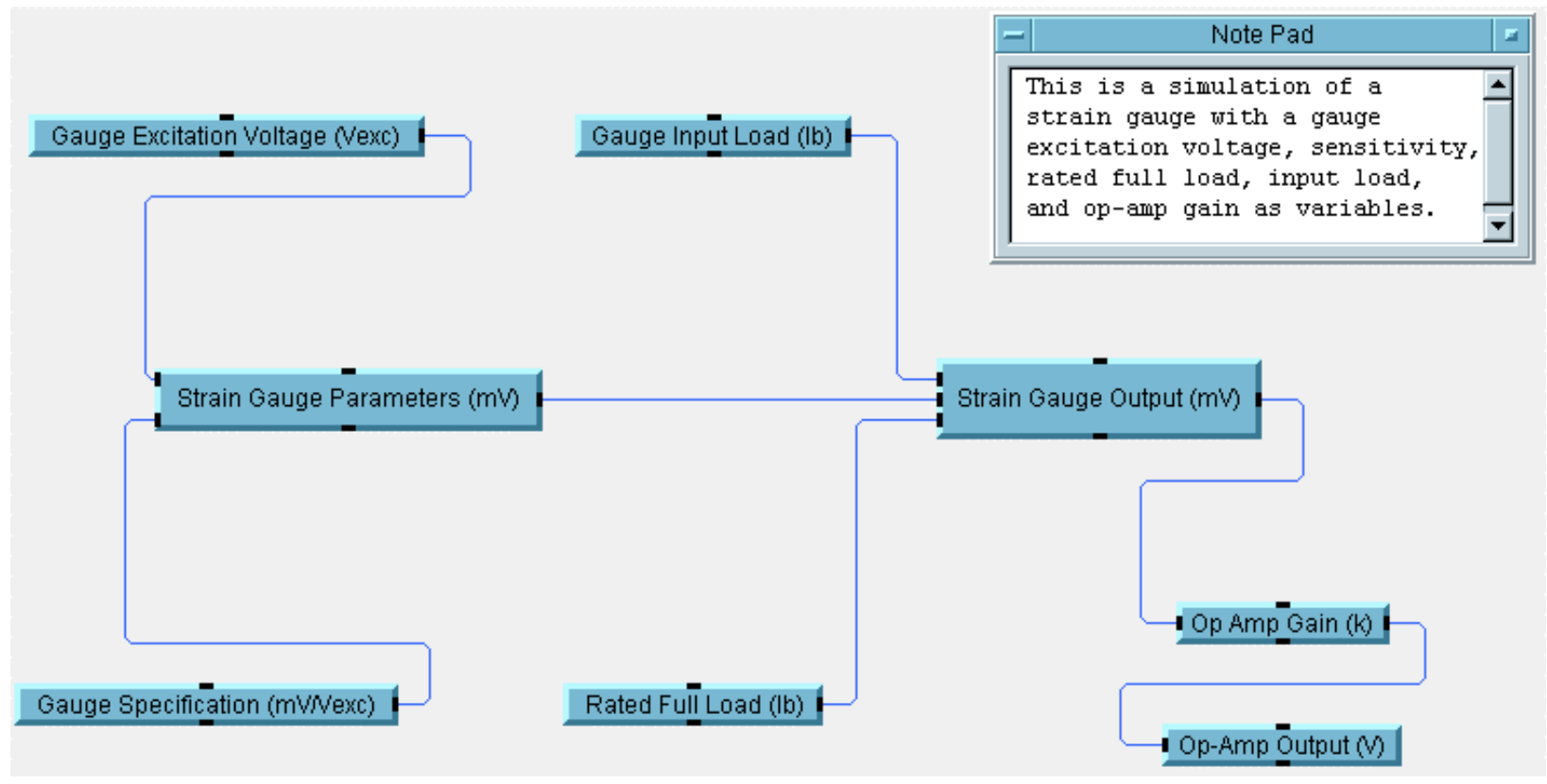

Figure 2 VEE Pro Program for Strain Gage Simulation with Closed Icons

The objective is to analyze the systems by opening the icons and understanding the measurement system; they alter the parameters and observe the results. The program simulates a strain gage with excitation voltage, sensitivity, rated full load, input load, and op-amp gain as variables. The outcome illustrates VEE Pro's capabilities and limitations. The attendees then experiment with different values of excitation voltage, input load, specifications, rated full load, and op amp gain.

Proceedings of the 2001 American Society for Engineering Education Annual Conference \& Exposition Copyright 2001, American Society for Engineering Education 


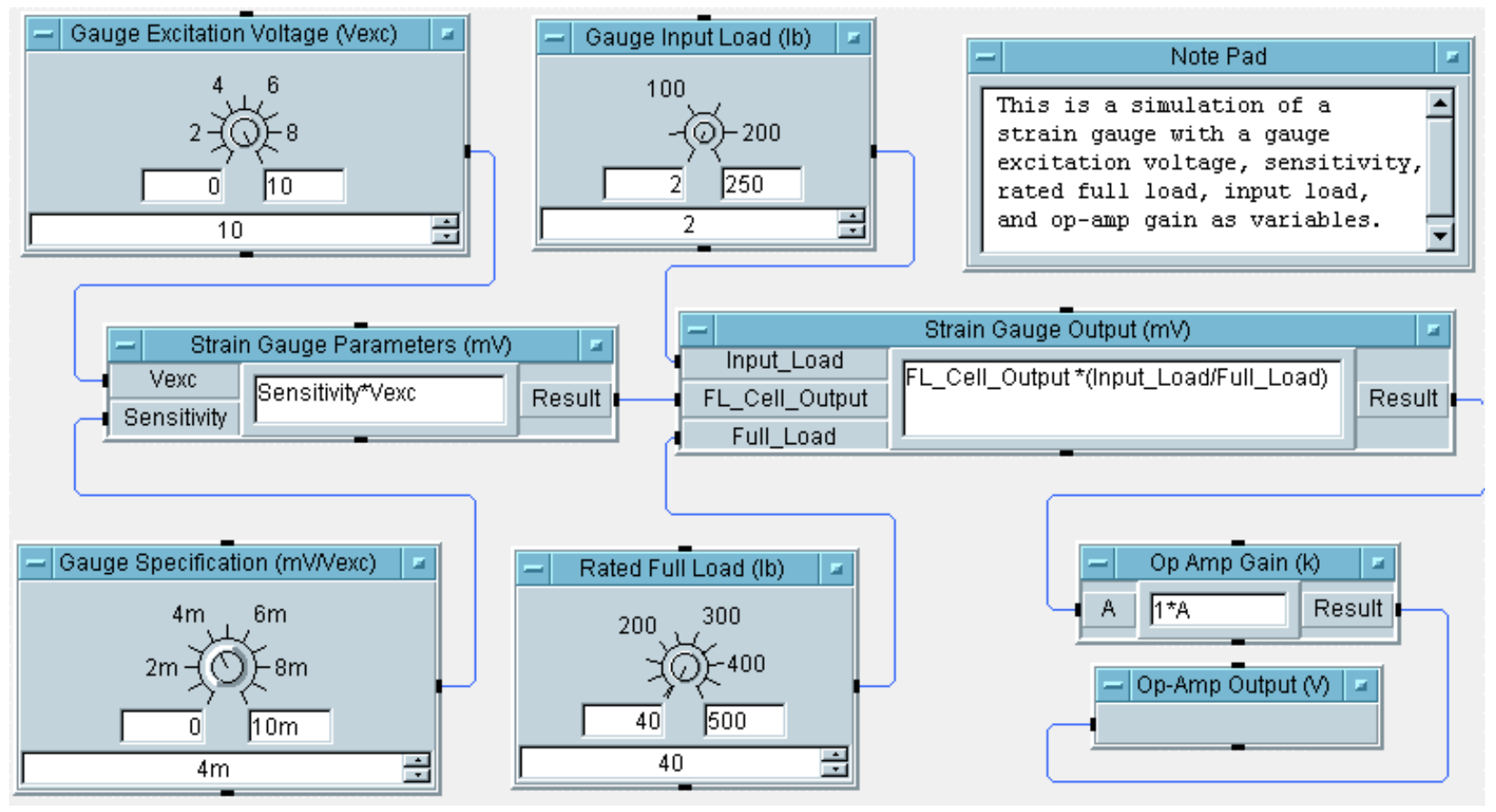

Figure 3 Program for Strain Gage Simulation with Open Icons

Figure 3 shows the program above with icons open. This representation shows what happens inside each function, equipment, or process. For example, the excitation voltage, input load, gage specifications and rated full load can be varied with a knob. The outputs are viewed in the op amp gain and output boxes on the lower right side. Minimal emphasis is on the programming; concentration is to enable better communication among technical personnel. Managers maintain focus on results; support staff develop relevant, productive systems to get timely results.

In summary, data acquisition systems are evaluated bottom up. Therefore, engineers and managers understand the operation of VEE-based systems in the operational mode. In the development mode, they manipulate a series of examples beginning with closed icons for an overview of the process or test. Opening the icons, varying parameters, and functionality of the test or process familiarizes participants with capabilities and limitations of the software.

\section{Review of Course Evaluation by Corporate Education}

The first offering of the "Inside the Icons" course was evaluated using the Northeastern University Corporate Education Continuing Education Evaluation/Quality Assurance Form. The results are summarized below. 
The first section evaluated course design and educational materials. The time to achieve the course objectives, organization and handouts, time for discussion and questions, and appropriate texts averaged "agree" on a five step scale ranging from "strongly disagree" to "strongly agree."

Student comments were that teachers were "good engineers and answered questions well." However, they should "teach more rather than self-paced." Another said that the focus should be "on applications of various tools within VEE and not so much on changing numbers and looking at wave theory."

Comments regarding the instructor included: "more teaching and less reading" and "gave basic overview; more teaching would be better."

The overall evaluation was rated from fair to excellent. The average was "Good." Comments included: "more teacher input would be better" and "do overview outside the lesson and have more detailed labs during the lesson."

The teaching experience indicated that four out of five achieved the objectives, and three out of four would recommend the course to others.

\section{Lessons Learned in Developing Engineering and Engineering Technology Laboratories}

The experience gained from teaching this software package to technicians and technologists in industry, and then to practicing engineers, has provided valuable insight into developing this type of laboratory for undergraduate students. The intent is to share our findings in the hope that your labs will gain from our experience. Two of the producers of test and measurement software described in the paper issue regular newsletters and bulletins for the academic community ${ }^{1,5}$. These publications describe special offers to academics to use the software for development and use in educational and industrial laboratories. They also publish experiences of and applications from faculty in using the software. Two recent publications are cited in the references.

Since the software is graphic and easy to understand, the opportunity to exploit the capabilities in the laboratory are exciting. One of the barriers to distance learning and off-site programs in engineering and technology education have been delivering labs. These software packages providing virtual labs and the power of the Internet provide the opportunity. Two of the numerous articles on e-learning and virtual labs show examples of the power of the technology.

The article on e-learning ${ }^{8}$ describes the components of the system necessary for a remote classroom in the year 2005. It is reported that University of Maryland's University College in Adelphi has an enrollment of 30,000. In $19985 \%$ of higher education students were involved in e-learning; it is projected to increase to $15 \%$ in 2002. ASEE's Distance Learning and Continuing Education catalog lists over 3000 courses. As technology continues to develop and expand, the opportunities for off-site and distance learning will expand. Students will be 
provided yet another alternative to learn the right knowledge at the right time. Now the opportunity for labs to be delivered remotely becomes a real alternative.

Web-based labs are available and are cost effective but, most important, students learn and experience reality. Leading engineering programs at MIT, Illinois Tech, College of Mines at University of Arizona have examples of labs on the Internet. Software packages mentioned in this paper and the power of computer technology drives this effort. It is the consensus that real labs will never be replaced; however, the capability broadens the ability for students to participate and acquire the full breadth of a technically based curricula. "The virtual lab will never be able to replicate the unexpected characteristics of a physical laboratory-the weird noise, the random error, the faulty machinery. And there's no simulating, say, the feel of a sediment, the heaviness of the weight, [or] the turn of a screw. Some things must be experienced first hand." $" 8$

\section{Summary}

This paper has described the development of a unique technique to enable engineers to communicate with the technical workforce on the utilization of VEE Pro software without knowing the details of its programming. Two other packages are qualitatively summarized. An example of how the material is presented is shown and described in the paper.

With this background, the authors present the basis for development of a computer-based, remote delivery of laboratories. The virtual lab will never totally replace the real lab. However, today's technology allows us to reach a broader audience without compromising the educational objectives. The virtual labs can be delivered as developed or transferred to real instruments through data acquisition cards.

The possibilities are without bounds. Our engineering background, education and training will lead us to new opportunities.

\section{Bibliography}

1. Engineering Educator, Agilent Technologies, Volume 4, Number 3, November 2000.

2. Hansberry, E. W., Hulbert, T. E., and Angus R. B., Using Concurrent Engineering and Just-In-Time Education $^{\mathrm{TM}}$ Techniques to Develop Technician Retraining, 33rd ASEE Midwest Section Conference on Hands-On Engineering, Salina, KS, April 8-10, 1998.

3. Hulbert, T. E. and Angus, R. B., Development and Delivery of Data Acquisition and HPVEE Courses for Technical Personnel, ASEE Conference Proceedings, St. Louis, MO. June 2000.

Proceedings of the 2001 American Society for Engineering Education Annual Conference \& Exposition Copyright 2001, American Society for Engineering Education 
4. Hulbert, T. E., Hansberry, E. W., and Angus, R. B., Just-In-Time Education ${ }^{\mathrm{TM}}$ : An Idea WhoseTime is Overdue,ASEE Annual Conference Proceedings, June 1996.

5. Instrumentation Newsletter, National Instruments, Fourth Quarter, 2000.

6. Mager, R. F., Preparing Instructional Objectives, Fearon Publishers, 1962.

7. Mannix, Margaret The Virtues of Virtual Labs, ASEE Prism, September, 2000.

8. Ubell, Robert, Engineers Turn to E-Learning, IEEE Spectrum, October, 2000.

\section{Biographies}

Thomas E. Hulbert is a Registered Professional Engineer and Professor Emeritus at Northeastern University. For 33 years, he served as a faculty member and administrator in the College of Engineering. For the last 13 years, he was Director and Associate Dean of the School of Engineering Technology. Prior to joining Northeastern, he worked as a Senior Industrial Engineer for 8 years. He has also consulted in the areas of facilities design and inventory control. He has written numerous papers on educational innovation and has contributed to several textbooks. He currently coordinates the Professional Engineering Review Courses at Northeastern University.

Robert B. Angus is a Senior Lecturer at Northeastern University with 53 years of teaching experience covering mathematics, physics, and electrical engineering courses. He has authored or co-authored twelve textbooks and numerous technical papers. He has also been an engineer, engineering manager, and senior engineering specialist for more than 20 years. In addition, he is an engineering consultant for 30 years specializing in circuit and system design, curriculum development, and technical manual writing. He is currently writing a family of Circuits texts and manuals to be used in an integrated course developed using Just-in-Time Education ${ }^{\mathrm{TM}}$ concepts.

Proceedings of the 2001 American Society for Engineering Education Annual Conference \& Exposition

Copyright 2001, American Society for Engineering Education 